\title{
Guía de práctica clínica para el tratamiento farmacológico y psicológico de los pacientes adultos con depresión y un diagnóstico comórbido de trastorno por uso de sustancias
}

\author{
Clinical practice guideline on pharmacological \\ and psychological management of adult patients with \\ depression and a comorbid substance use disorder
}

\author{
Marta Torrens ${ }^{1 *}$, Judit Tirado-Muñoz ${ }^{1 *}$, Francina Fonseca*, Magi Farré ${ }^{1 * *}$, \\ Ana González-Pinto***, Manuel Arrojo****, Miquel Bernardo*****, Belén Arranz******, \\ Marina Garriga*****, Pilar A. Sáiz*******, Gerardo Flórez********, José Manuel \\ Goikolea*****, Iñaki Zorrilla***, Ruth Cunill******, Xavier Castells*********, \\ Elisardo Becoña**********, Ana López**********, Luis SAN******. \\ ${ }^{1}$ Estos autores han contribuido por igual a este trabajo. \\ * Grupo de Investigación en Adicciones. IMIM-Institut Hospital del Mar d'Investigacions Mèdiques, Barcelona, España. \\ ** Unidad de farmacología clínica. Hospital Universitari Germans Trias i Pujol, Institut de Recerca Germans Trias (HUGTP- \\ IGTP), Badalona y Universitat Autònoma de Barcelona (UAB), RTA, Barcelona, España. \\ *** Instituto de Investigación Sanitaria BIOARABA. OSI Araba. Hospital Universitario. CIBERSAM, UPV/EHU, Vitoria, España. \\ **** Servicio de Psiquiatría. EOXI de Santiago de Compostela, España. \\ ***** Hospital Clinic, Institut d'Investigacions Biomèdiques August Pi i Sunyer (IDIBAPS), CIBERSAM, Barcelona, España. \\ ****** Parc Sanitari Sant Joan de Deu. CIBERSAM, Barcelona, España. \\ ******** Universidad de Oviedo, CIBERSAM, Instituto de Investigación Sanitaria del Principado de Asturias (ISPA), Servicio de \\ Salud del Principado de Asturias (SESPA), Oviedo, España. \\ ******** Unidad de Conductas Adictivas, Complejo Hospitalario de Ourense. CIBERSAM, Ourense, España. \\ ********* Grupo de investigación TransLab, Departamento de Ciencias Médicas. Universitat de Girona, España. \\ ********** Unidad de Tabaquismo y Trastornos Adictivos, Facultad de Psicología. Universidad de Santiago de Compostela, \\ Santiago de Compostela, España.
}

\section{Resumen}

La concurrencia de depresión y un trastorno por uso de sustancias (TUS) en pacientes que presentan patología dual ha sido reconocida desde hace mucho tiempo como una consideración importante en la práctica clínica. Esta revisión sintetiza la evidencia de intervenciones farmacológicas y psicosociales para trastornos comórbidos de depresión y uso de sustancias y además proporciona recomendaciones clínicas respecto de las mejores intervenciones para tratar a estos pacientes. Se utilizó la mejor evidencia de ensayos controlados aleatorizados para evaluar las opciones de tratamiento. La fuerza de las recomendaciones se describió mediante el enfoque GRADE. Nuestros resultados sugieren que: 1) en pacientes con depresión y consumo de

\begin{abstract}
Co-occurrence of depression and a substance use disorder (SUD) in patients who present dual diagnoses has been long recognized as an important consideration in clinical practice. This review synthesizes the evidence of pharmacological and psychosocial interventions for comorbid depressive disorders and SUDs while providing clinical recommendations about the best interventions to address these patients. The best evidence from randomized controlled trials was used to evaluate treatment options. The strength of recommendations was described using the GRADE approach. Our results suggest that 1) In patients with depression and alcohol consumption, the administration of non-selective serotonin reuptake inhibitor (SSRI)
\end{abstract}


alcohol, se recomienda la administración de antidepresivos inhibidores de la recaptación de serotonina (ISRS) no selectivos en lugar de los ISRS para mejorar los síntomas depresivos (recomendación fuerte). No se recomiendan antidepresivos ISRS (recomendación fuerte) ni antidepresivos no ISRS (recomendación débil) para reducir el consumo de alcohol; 2) en pacientes con depresión y consumo de cannabis, no se recomienda el uso de venlafaxina (recomendación débil); 3) en pacientes con depresión y consumo de cocaína, no se recomienda el uso de antidepresivos ISRS para mejorar los síntomas depresivos (recomendación débil) o para reducir el consumo de cocaína (recomendación fuerte). El uso de antidepresivos no ISRS solo se recomienda para mejorar los síntomas depresivos (recomendación fuerte); 4) no se recomienda la administración de bupropión para reducir el consumo de nicotina (recomendación fuerte), y 5 ) en cuanto al tratamiento psicológico, en pacientes con depresión y trastorno de alcohol concurrente, tanto la farmacoterapia como la terapia cognitivoconductual tienen efectos positivos en la internalización de los síntomas y en la reducción del consumo de alcohol (recomendación débil). Nuestra revisión sugiere la necesidad de realizar más investigaciones en esta área y de estudios aleatorizados, multisitio y más grandes para proporcionar más evidencia definitiva.

Palabras clave: Depresión; trastorno por uso de sustancias; alcohol; cocaína; cannabis; nicotina; antidepresivos; inhibidores selectivos de la recaptación de serotonina.

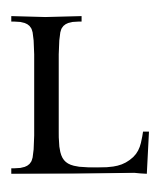
a concurrencia de depresión y un trastorno por uso de sustancias (TUS) en pacientes que presentan patología dual ha sido reconocida desde hace mucho tiempo como una consideración importante en la práctica clínica (Iqbal, Levin y Levin, 2019). Esta fuerte asociación entre TUS y depresión mayor ha sido confirmado en varios metaanálisis de estudios epidemiológicos publicados entre 1990 y 2014 (razón de momios [RM] 3,80) (Lai, Cleary, Sitharthan y Hunt, 2015) y entre 1990 y 2019 (Hunt, Malhi, Lai y Cleary, 2020). Los estudios coinciden en una prevalencia de la depresión en un rango desde el $15 \%$ en la población general hasta el $80 \%$ en una selección basada en otros criterios de muestras clínicas tanto de entornos de salud mental como de entornos relacionados con las adicciones (Caetano, Vaeth y Canino, 2019; Compton, Thomas, Stinson y Grant, 2007; Torrens, Rossi, Martínez-Riera, Martínez-Sanvisens y Bulbena, 2012; Vázquez, Torres, Otero y Díaz, 2011). Por sustancias, la comorbilidad entre depresión mayor y TUS tiene una prevalencia de $20 \%$ para el trastorno por uso de alcohol (TUA) (Boschloo et al., 2011; Carton et al., 2018), entre el $16 \%$ y el $34 \%$ para el uso de cocaína (Alías-Ferri et al., 2021; Vergara-Moragues et al., 2012) y entre $13,5 \%$ y $38 \%$ para trastorno por uso de cannabis (Cuenca-Royo, Torrens, Sánchez-Niubó, Suelves y Domingo-Salvany, 2013) antidepressants instead of SSRI is recommended for improvement of depressive symptoms (strong recommendation). Neither SSRI (strong recommendation) nor non-SSRI (weak recommendation) antidepressants are recommended for reduction in alcohol consumption. 2) In patients with depression and cannabis use, the use of venlafaxine is not recommended (weak recommendation). 3) In patients with depression and cocaine consumption, the use of SSRI antidepressants for improving depressive symptoms (weak recommendation) or to reduce cocaine use is not recommended (strong recommendation). The use of non-SSRI antidepressants is only recommended for improving depressive symptoms (strong recommendation). 4) The administration of bupropion to reduce nicotine consumption is not recommended (strong recommendation). 5) Regarding psychological treatment, in patients with depression and co-occurring alcohol disorder, both pharmacotherapy and cognitive behavioural therapy have positive effects on internalizing symptoms and in reducing alcohol consumption (weak recommendation). Our review suggests the need for more research in this area and for larger, multisite, randomized studies to provide more definite evidence.

Keywords: Depression; substance use disorder; alcohol; cocaine; cannabis; nicotine; antidepressants; selective serotonin reuptake inhibitors.

y entre $43,2 \%$ y $61,2 \%$ para el uso de tabaco (Jiménez-Treviño et al., 2019). Según estudios epidemiológicos y clínicos, la depresión mayor comórbida es dos veces más frecuente en mujeres con TUS que en la población general y la presencia de esta comorbilidad es más frecuente en las mujeres que en los hombres (Farré, Tirado-Muñoz y Torrens, 2017; Tirado-Muñoz, Farré, Mestre-Pintó, Szerman y Torrens, 2018).

La comorbilidad entre depresión y TUS puede ser explicada en términos causales, con la presencia de un trastorno aumentando la probabilidad de la ocurrencia del otro (Khantzian, 1985). La comorbilidad también podría ser causada por el uso de sustancias que revela una predisposición latente a la depresión en individuos de alto riesgo (Schuckit, 2006). Por último, los factores comunes predisponentes, como la exposición a factores biológicos, sociales o ambientales a eventos vitales adversos tempranos en la forma de maltrato y/o negligencia infantil pueden aumentar la probabilidad de depresión y TUS (Rappeneau y Bérod, 2017).

En comparación con pacientes con un solo trastorno, la comorbilidad de depresión y TUS comúnmente se asocia con un diagnóstico inexacto, el empeoramiento de la evolución clínica, un mayor deterioro funcional, una menor adherencia a la medicación, más recaídas en el uso de 
sustancias, así como un mayor riesgo de conducta suicida (Torrens et al., 2011; Torrens et al., 2012). Además, los profesionales de la salud mental y del tratamiento del TUS se enfrentan a las dificultades de brindar asistencia eficaz a los pacientes cuyos problemas se solapan entre dos especialidades de atención sanitaria. En este sentido, el tratamiento del TUS debe integrarse en las estrategias de manejo de los trastornos depresivos no relacionados con el TUS, combinando intervenciones farmacológicas y psicoterapéuticas adecuadas (Tirado-Muñoz et al., 2018). La cuidadosa selección del tratamiento farmacológico que evalúe la eficacia, la seguridad, las posibles interacciones farmacológicas y los problemas relacionados con la adherencia es crucial en pacientes con trastorno depresivo y TUS. Hasta la actualidad, las revisiones y los metaanálisis sistemáticos han informado los resultados de las intervenciones en pacientes con TUS concurrente y trastorno del estado de ánimo (Agabio, Trogu y Pani, 2018; Carey, 2019; Conner, Pinquart y Holbrook, 2008; Conner, Pinquart y Gamble, 2009; Tirado-Muñoz et al., 2018; Torrens, Fonseca, Mateu y Farre, 2005).

Esta revisión sintetiza las intervenciones farmacológicas y psicosociales que se han realizado en el trastorno depresivo comórbido y el uso concurrente de alcohol, cocaína, nicotina o cannabis. También proporciona a los profesionales de la salud involucrados en el cuidado de estos pacientes unas recomendaciones clínicas basadas en evidencia científica para ayudar en el proceso de toma de decisiones en su práctica clínica.

\section{Métodos}

\section{Formulación de preguntas clínicas}

De acuerdo con los principios de la medicina basada en la evidencia, utilizamos la estructura PICO (Paciente-Intervención-Comparación-Outcome/resultados) (Guyatt et al., 2011; Schünemann et al., 2008) para formular la siguiente pregunta de revisión: «¿Cuál es el efecto de una intervención farmacológica y/o psicológica para el tratamiento de pacientes adultos con depresión y un TUS?». La población objetivo de esta guía clínica era los pacientes mayores de 18 años diagnosticados con una depresión y un TUS (incluidos cannabis, cocaína, alcohol y/o nicotina). No se incluyó en esta revisión el trastorno por consumo de opioides porque no se identificaron revisiones sistemáticas con o sin metaanálisis o ensayos clínicos aleatorizados.

\section{Estrategia de búsqueda}

Las búsquedas de estudios relevantes publicados hasta diciembre de 2015 se hicieron en las bases de datos MEDLINE, PsycINFO, Embase, Scopus, Web of Science, Cochrane Library y Pubmed con una búsqueda de actualizaciones hasta mayo de 2016. La Tabla 1 describe la estrategia de búsqueda y los diferentes términos utilizados.

\section{Criterios de elegibilidad}

Los estudios fueron elegibles para su inclusión si 1) eran metaanálisis, revisiones Cochrane, revisiones sistemáticas o ensayos clínicos (aleatorizado, doble ciego y controlado con placebo) de cualquier tratamiento farmacológico y

Tabla 1. Descripción de los términos de la búsqueda.

\begin{tabular}{|c|c|c|}
\hline Tema & Palabras clave & Limitaciones aplicadas \\
\hline Depresión & $\begin{array}{l}\text { dysthymic disorder; depressive disorder; depressive disorder, major; } \\
\text { mood disorders }\end{array}$ & \multirow{11}{*}{$\begin{array}{l}\text { Human, } 18 \text { years or older, } \\
\text { RCT OR Review, Systematic } \\
\text { Reviews, Meta-Analysis }\end{array}$} \\
\hline Comorbilidad & $\begin{array}{l}\text { substance abuse, substance dependence, substance use, comorbidity, } \\
\text { misuse, co-occurr*, coexist*, concurren* dual diagnosis dual disorder, } \\
\text { dual pathology }\end{array}$ & \\
\hline Alcohol & alcohol drinking, drinking behavior, alcohol use, alcohol abuse & \\
\hline Cocaína & cocaine-related disorders, cocaine use, cocaine abuse & \\
\hline Cannabis & cannabis use, marijuana Abuse marijuana Smoking & \\
\hline Nicotina & nicotine use & \\
\hline Tratamiento farmacológico (Antidepresivos) & antidepressive agents, tricyclic; tricyclic antidepressant, SSRI & \\
\hline Tratamiento farmacológico (..) & $\begin{array}{l}\text { oxcarbazepine, oxcarbazepine, carbamazepine, carbamazepine, } \\
\text { lamotrigine, lamotrigine, valproic acid, valproate, divalproex, lithium }\end{array}$ & \\
\hline Tratamiento farmacológico (...) & benzodiazepines & \\
\hline Tratamiento farmacológico (..) & $\begin{array}{l}\text { varenicline, nicotine replacement therapy, bupropion, topiramate, } \\
\text { acamprosate, naltrexone, anticraving, cyanamide, disulfiram, } \\
\text { antidipsotropic }\end{array}$ & \\
\hline Tratamiento psicológico & $\begin{array}{l}\text { behavioral therapy, therapy, cognitive therapy, social skills, contingency } \\
\text { management, time out, reinforcement programs, token economy, self- } \\
\text { help, motivational interview, mindfulness, cue exposure, self-control, } \\
\text { psychoeducation, psychotherapy }\end{array}$ & \\
\hline
\end{tabular}


psicológico; 2) los pacientes habían sido diagnosticados de un trastorno depresivo mayor y un trastorno por uso de sustancias (alcohol, cannabis, cocaína o nicotina), y 3) el resultado fue el uso de sustancias (disminución o abstinencia) y/o síntomas depresivos. Los estudios seleccionados incluyeron participantes con un solo TUS (alcohol, cocaína, cannabis o nicotina) según la sustancia de interés.

\section{Evaluación de la calidad de la evidencia y formulación de las recomendaciones}

La evaluación de la calidad de los estudios y el resumen de la evidencia para cada pregunta se realizó siguiendo las recomendaciones del grupo de trabajo GRADE (Grading of Recommendations Assessment, Development and Evaluation) (www.gradeworkinggroup.org) (Guyatt et al., 2008). Cada artículo se leyó en detalle y se evaluó críticamente de acuerdo con GRADE, luego se discutió entre los autores, lo que resultó en una puntuación general de evaluación de calidad, posteriormente revisada por resultado individual. Todo el proceso culminó en una recomendación clínica que se calificó según su fuerza. En aras de la claridad, las recomendaciones se dividen aquí según la sustancia.

\section{Revisión y evaluación externas}

La evidencia se evaluó utilizando el instrumento AGREE II (Appraisal of Guidelines for Research and Evaluation) (Gopalakrishna, Langendam, Scholten, Bossuyt y Leeflang, 2013) (www.agreecollaboration.org).

Se puede encontrar información más detallada sobre la metodología en publicaciones anteriores (San y Arranz, 2016).

\section{Resultados}

La Figura 1 describe el diagrama de flujo PRISMA que resulta en la selección del estudio. La búsqueda produjo 741 estudios. Se consideraron elegibles para una evaluación adicional 84 estudios. La selección definitiva incluyó 32 estudios. No se incluyeron en la Guía los ensayos abiertos, los estudios de cohortes o de casos y controles, los estudios transversales y observacionales, los informes de casos,

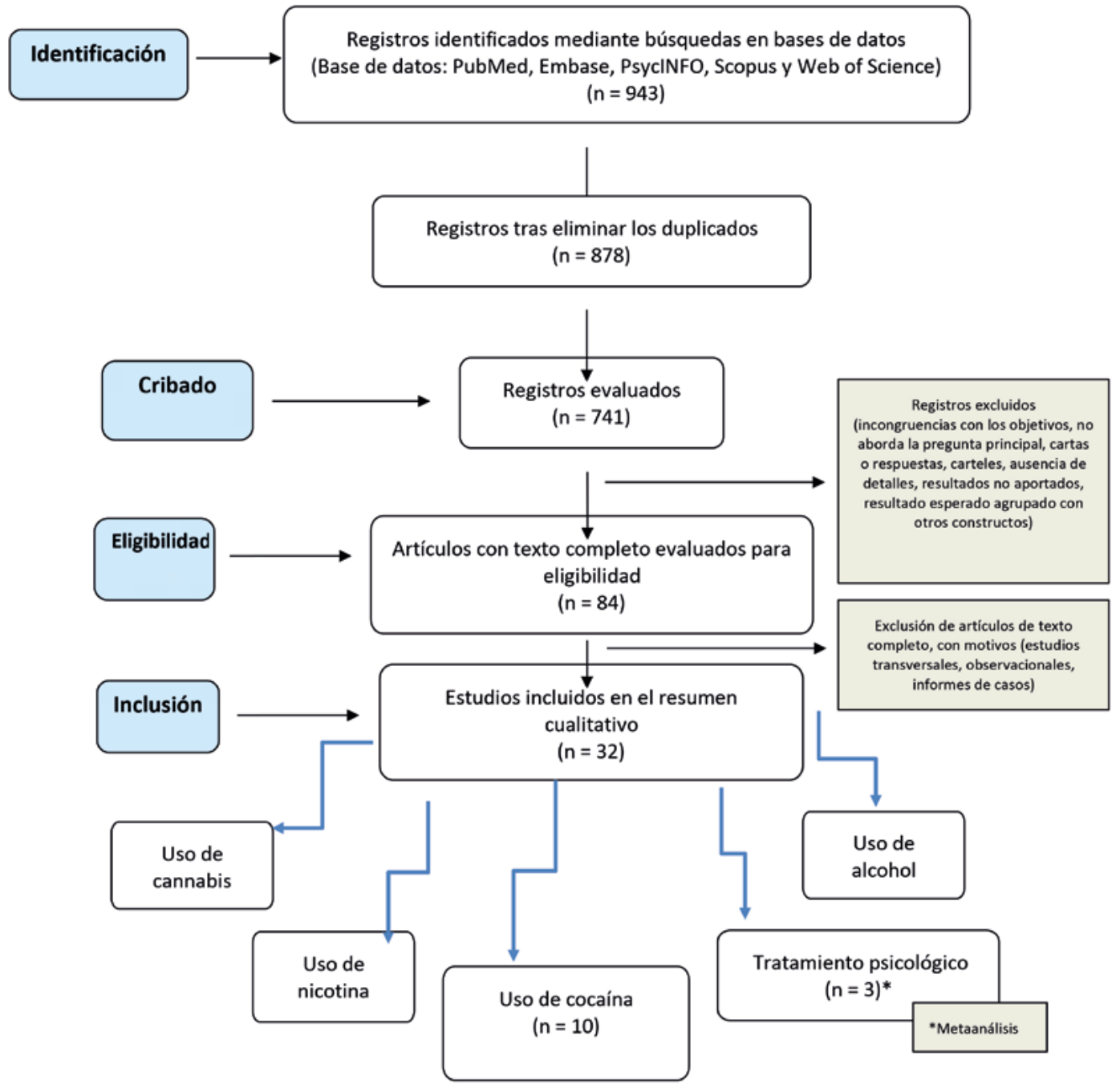

Figura 1. Diagrama de flujo del proceso de selección de estudios. 
las cartas, los carteles y los resúmenes de presentaciones en reuniones y conferencias de especialistas. Solo se incluyeron artículos publicados en inglés. Los datos se extrajeron de los estudios incluidos mediante una plantilla predefinida y la calidad de cada estudio se evaluó mediante criterios estándar. Las Tablas 2 a 5 presentan un informe resumido de estos estudios. Solo se incluyeron los metaanálisis para las intervenciones psicológicas.

\section{Pacientes con trastorno depresivo y uso de alcohol}

Los detalles sobre los estudios incluidos se muestran en la Tabla 2.

Pregunta 1 de PICO. ¿Es eficaz la administración de inhibidores selectivos de la recaptación de serotonina (los ISRS) para disminuir los síntomas de la depresión, reducir el consumo de alcohol o mejorar las variables pragmáticas y de funcionalidad en pacientes con depresión y consumo de alcohol?
Siete ECA evaluaron la eficacia de los ISRS (principalmente fluoxetina y sertralina) para reducir los síntomas depresivos (Cornelius et al., 1997; Gual et al., 2003; Kranzler et al., 2006; Moak et al., 2003; Pettinati et al., 2001, 2010; Roy, 1998). En seis ECA (Gual et al., 2003; Kranzler et al., 2006; Moak et al., 2003; Pettinati et al., 2001, 2010) no se encontraron diferencias entre ISRS y placebo a las 12 semanas utilizando la escala de calificación de depresión de Hamilton (HAMD) $(\mathrm{n}=498)$ (evidencia de baja calidad). Utilizando el Inventario de Depresión de Beck (BDI), tampoco se encontraron diferencias en cuatro ECA (Cornelius et al., 1997; Moak et al., 2003; Pettinati et al., 2001; Roy, 1998) $(\mathrm{n}=184)$ (evidencia de calidad moderada).

Cinco ECA se centraron en el consumo de alcohol como resultado de interés utilizando el método de calendario de seguimiento de línea de tiempo (TLFB) (Cornelius et al., 1997; Gual et al., 2003; Kranzler et al., 2006; Pettinati et al., 2001; Sobell y Sobell, 1992). En estos cinco ECA, no se encontraron diferencias entre los ISRS y el placebo $(n=431)$.

Tabla 2. Depresión y trastorno por uso de alcohol.

\begin{tabular}{|c|c|c|c|c|c|c|c|}
\hline AUTORES & $\begin{array}{l}\text { FÁRMACO } \\
\text { UTILIZADO } \\
\text { MG/DIA }\end{array}$ & $\begin{array}{l}\text { DIAGNÓSTICO/ } \\
\text { INSTRUMENTO }\end{array}$ & $\begin{array}{l}\text { CASO/ } \\
\text { CONTROL }\end{array}$ & $\begin{array}{l}\text { CONSUMO AL } \\
\text { INICIO DEL ECA }\end{array}$ & SEMANAS & $\begin{array}{c}\text { TERAPIA } \\
\text { CONCOMITANTE }\end{array}$ & VARIABLE DE RESULTADO \\
\hline $\begin{array}{l}\text { Altamura } \\
1990\end{array}$ & $\begin{array}{l}\text { Viloxazina (400) } \\
\text { Placebo }\end{array}$ & $\begin{array}{c}\text { DSM-III-R/NA } \\
\text { (distimia } \geq 18 \text { en } \\
\text { HRSD) }\end{array}$ & $14 / 13$ & $\begin{array}{c}7 \text { días de } \\
\text { abstinencia }\end{array}$ & 12 & $\begin{array}{l}4 \text { semanas de } \\
\text { ingreso seguidas } \\
\text { de tratamiento } \\
\text { ambulatorio }\end{array}$ & $\begin{array}{l}\text { TLFB: Ambos grupos mejoran el consumo de } \\
\text { alcohol sin diferencias significativas. } \\
\text { HAMD: Mejoría significativa en el grupo } \\
\text { viloxacina ( } 5 \text { vs. } 21, p<0,01 \text { ). }\end{array}$ \\
\hline $\begin{array}{l}\text { Mc Grath } \\
1996\end{array}$ & $\begin{array}{l}\text { Imipramina } \\
(150-300) \\
\text { Placebo }\end{array}$ & DSM-III-R/SCID & $27 / 29$ & Consumo actual & 12 & $\begin{array}{l}\text { TCC individual } \\
\text { y prevención de } \\
\text { recaídas }\end{array}$ & $\begin{array}{l}\text { TLFB: Ningún efecto general en el consumo de } \\
\text { alcohol ( } 4 \text { últimas semanas de abstinencia). } \\
\text { En los pacientes con mejoría del ánimo, el } \\
\text { consumo de alcohol disminuyó más en los } \\
\text { tratados con imipramina } \\
\text { HAMD: El grupo Imipramina mostró valores } \\
\text { significativamente más bajos }(9,4 \pm 7,7) \text { que el } \\
\text { grupo Placebo }(12,4 \pm 9,7)(p<0,03) \text {. }\end{array}$ \\
\hline Mason 1996 & $\begin{array}{l}\text { Desipramina (200) } \\
\text { Placebo }\end{array}$ & DSM-III-R/NA & $12 / 10$ & $\begin{array}{l}\geq 7 \text { días de } \\
\text { abstinencia }\end{array}$ & 24 & $\begin{array}{l}\text { Alcohólicos } \\
\text { anónimos }\end{array}$ & $\begin{array}{l}\text { TLFB: Los pacientes con desipramina } \\
\text { mostraron un periodo más largo de } \\
\text { abstinencia que el grupo P (media: } 109 \text { frente } \\
\text { a } 65 \text { días) ( } p=0,03 \text { ) } \\
\text { HAMD: El grupo con desipramina disminuyó } \\
\text { las puntuaciones significativamente, con } \\
\text { respecto al Placebo (media: } 8 \text { vs. } 20 \text { ) ( } p \\
<0,01 \text { ). }\end{array}$ \\
\hline $\begin{array}{l}\text { Cornelius } \\
1997\end{array}$ & $\begin{array}{l}\text { Fluoxetina (20) } \\
\text { Placebo }\end{array}$ & DSM-III-R/SCID & $25 / 26$ & $\begin{array}{l}\geq 9 \text { días de } \\
\text { abstinencia }\end{array}$ & 12 & $\begin{array}{c}\text { Psicoterapia de } \\
\text { apoyo }\end{array}$ & $\begin{array}{l}\text { TLFBI: El consumo de alcohol total fue } \\
\text { significativamente menor en el grupo } \\
\text { fluoxetina que en el grupo placebo. } \\
\text { HAMD: Mejoría significativa en los síntomas } \\
\text { depresivos en el grupo fluoxetina en } \\
\text { comparación al grupo placebo. }\end{array}$ \\
\hline Roy 1998 & $\begin{array}{l}\text { Sertralina (100) } \\
\text { Placebo }\end{array}$ & DSM-III-R/NA & $10 / 5$ & $\begin{array}{l}\geq 14 \text { días de } \\
\text { abstinencia }\end{array}$ & 6 & $\begin{array}{l}\text { Ingreso seguido } \\
\text { de hospital de día } \\
\text { intensivo }\end{array}$ & $\begin{array}{l}\text { Consumo: No evaluado } \\
\text { BDI, HAMD: el grupo sertralina mostró } \\
\text { puntuaciones significativamente más bajas en } \\
\text { HAMD }(12,7 \pm 9,1) \text { y en BDI }(18,5 \pm 12,5) \text { en } \\
\text { comparación a placebo }(16,3 \pm 7,5 \text { y } 23,1 \pm \\
10,2) \text { ( } p<0,003 \text { y } p<0,03) .\end{array}$ \\
\hline $\begin{array}{l}\text { Roy-Byrne } \\
2000\end{array}$ & $\begin{array}{l}\text { Nefazodona }(460 \\
\pm 75) \\
\text { Placebo }\end{array}$ & DSM-III-R/SCID & $20 / 11$ & Consumo actual & 12 & TCC grupo & $\begin{array}{l}\text { TLFBI: disminución no significativa en el } \\
\text { número medio de bebidas alcohólicas diarias } \\
\text { en nefazodona (3) vs. grupo } P(4) \\
\text { HAMD: nefazodona mostró una mejora } \\
\text { significativa (12) en comparación al Placebo } \\
\text { (16) ( }<<0,1) \text {. }\end{array}$ \\
\hline
\end{tabular}


Marta Torrens, Judit Tirado-Muñoz, Francina Fonseca, Magi Farré, Ana González-Pinto, Manuel Arrojo, Miquel Bernardo, Belén Arranz, Marina Garriga, Pilar A Sáiz, Gerardo Flórez, José Manuel Goikolea, Iñaki Zorrilla, Ruth Cunill, Xavier Castells, Elisardo Becoña, Ana López, Luis San

\begin{tabular}{|c|c|c|c|c|c|c|c|}
\hline AUTORES & $\begin{array}{c}\text { FÁRMACO } \\
\text { UTILIZADO } \\
\text { MG/DIA }\end{array}$ & $\begin{array}{l}\text { DIAGNÓSTICO/ } \\
\text { INSTRUMENTO }\end{array}$ & $\begin{array}{c}\text { CASO/ } \\
\text { CONTROL }\end{array}$ & $\begin{array}{l}\text { CONSUMO AL } \\
\text { INICIO DEL ECA }\end{array}$ & SEMANAS & $\begin{array}{c}\text { TERAPIA } \\
\text { CONCOMITANTE }\end{array}$ & VARIABLE DE RESULTADO \\
\hline $\begin{array}{l}\text { Pettinati } \\
2001\end{array}$ & $\begin{array}{l}\text { Sertralina (200) } \\
\text { Placebo }\end{array}$ & DSM-III-R/SCID & $12 / 17$ & $\begin{array}{l}\geq 3 \text { días de } \\
\text { abstinencia }\end{array}$ & 14 & $\begin{array}{l}\text { Terapia de los } \\
\text { 12-pasos }\end{array}$ & $\begin{array}{l}\text { TLFB: diferencias no significativas en la } \\
\text { disminución del \% de los días bebiendo y } \\
\text { semanas hasta la recaída entre la sertralina y } \\
\text { el grupo Placebo } \\
\text { BDI, HAMD: diferencias no significativas en la } \\
\text { disminución en la HAMD y las puntuaciones } \\
\text { del BDI entre los grupos sertralina }(6,8 \text { y } 7,2 \\
\text { respectivamente) y Placebo }(8,8 \text { y } 9,1) \text {. }\end{array}$ \\
\hline
\end{tabular}

\begin{tabular}{lllllll}
\hline Gual 2003 & $\begin{array}{l}\text { Sertralina (50-150) } \\
\text { Placebo }\end{array}$ & DSM-IV/ NA & $24 / 22$ & $\begin{array}{l}\geq 14 \text { días de } \\
\text { abstinencia }\end{array}$ & 24 & $\begin{array}{c}2 \text { semanas de } \\
\text { abstinencia } \\
\text { después de la } \\
\text { desintoxicación }\end{array}$
\end{tabular}

Diferencias no significativas en las tasas de recaída en grupo sertralina $(31,8 \%)$ frente a grupo Placebo $(23,1 \%)$.

HAMD / MADRS: diferencias no significativas en las tasas de respuesta entre el grupo sertralina (44\%) y el grupo Placebo (39\%). Cuando los pacientes fueron estratificados en depresión grave (MADRS $\geq 26$ ) y moderada (MADRS <26), se observó un beneficio significativo del tratamiento con sertralina en el primer grupo.

\begin{tabular}{|c|c|c|c|c|c|c|c|}
\hline Moak 2003 & $\begin{array}{l}\text { Sertralina (186) } \\
\text { Placebo }\end{array}$ & DSM-III-R/PRISM & $38 / 44$ & $\begin{array}{l}\geq 3 \text { días de } \\
\text { abstinencia }\end{array}$ & 12 & $\begin{array}{l}\text { TCC individual } \\
\text { para alcohol y la } \\
\text { depresión }\end{array}$ & $\begin{array}{l}\text { TLFB: En el grupo sertralina menos bebidas por } \\
\text { día de consumo que el grupo Placebo ( } 2,3 \text { vs } \\
3,5, p=0,027) \text {. No otras diferencias. } \\
\text { HAMD, BDI: Menos depresión en las mujeres } \\
\text { tratadas con sertralina: } \mathrm{HAMD}=6,9 \text { vs } 9,3 \text { ( } p= \\
0,041 \text { ) y en } B D I=7,9 \text { vs } 10,4(p=0,005) \text { que } \\
\text { el grupo placebo. }\end{array}$ \\
\hline $\begin{array}{l}\text { Hernández- } \\
\text { Àvila } \\
2004\end{array}$ & $\begin{array}{l}\text { Nefazodona (200- } \\
600) \\
\text { Placebo }\end{array}$ & DSM-IV / SCID & $21 / 20$ & $\begin{array}{l}\geq 18 \text { bebidas/ } \\
\text { semana en } \\
\text { hombres o } 14 \\
\text { bebidas/ semana } \\
\text { mujeres }\end{array}$ & 10 & $\begin{array}{l}\text { Psicoterapia de } \\
\text { apoyo }\end{array}$ & $\begin{array}{l}\text { TLFB: Más sujetos tratados con nefazodona } \\
\text { ( } n=7 ; 33,3 \%) \text { eran abstinentes, en } \\
\text { comparación a los sujetos tratados con } \\
\text { placebo }(n=3 ; 15,0 \%) \text {. No significación } \\
\text { estadística ( } p=0,17) \text {. } \\
\text { HAMD: No hay diferencias entre los grupos ( } p \\
=0,82 \text { ). }\end{array}$ \\
\hline \multirow[t]{2}{*}{$\begin{array}{l}\text { Kranzler } \\
2006\end{array}$} & $\begin{array}{l}\text { Sertralina (200) } \\
\text { Placebo }\end{array}$ & $\begin{array}{l}\text { DSM-IV / PRISM } \\
\text { Grupo A*: } \\
\text { HDRS } \geq 17\end{array}$ & $89 / 100$ & $\begin{array}{l}\geq 18 \text { bebidas/ } \\
\text { semana en } \\
\text { hombres o } 14 \\
\text { bebidas/ semana } \\
\text { mujeres }\end{array}$ & 10 & No reportado & $\begin{array}{l}\text { TLFB/ HAMD: Tanto el consumo de alcohol } \\
\text { como los síntomas depresivos disminuyeron } \\
\text { sustancialmente con el tiempo en ambos } \\
\text { grupos. No hubo diferencias entre los grupos. }\end{array}$ \\
\hline & & $\begin{array}{l}\text { Grupo } B^{\star} \text { : } \\
\text { HDRS } \leq 16\end{array}$ & $70 / 69$ & & & & \\
\hline $\begin{array}{l}\text { Cornelius } \\
2009\end{array}$ & $\begin{array}{l}\text { Fluoxetina (20) } \\
\text { Placebo }\end{array}$ & $\begin{array}{l}\text { DSM-IV/ K-SADS- } \\
\text { PL+ SCID }\end{array}$ & $24 / 26$ & $\begin{array}{l}\text { Al menos } 10 \\
\text { bebidas durante } \\
\text { el mes previo a la } \\
\text { evaluación basal }\end{array}$ & 12 & $\begin{array}{l}\text { TCC y terapia } \\
\text { motivacional }\end{array}$ & $\begin{array}{l}\text { TLFB: Los sujetos del grupo fluoxetina y del } \\
\text { grupo placebo mostraron una disminución } \\
\text { significativa del consumo de alcohol. } \\
\text { HAMD: Los sujetos del grupo de fluoxetina } \\
\text { y del grupo placebo mostraron una mejora } \\
\text { significativa de los síntomas depresivos. }\end{array}$ \\
\hline $\begin{array}{l}\text { Pettinati } \\
2010\end{array}$ & $\begin{array}{l}\text { Sertralina }(200) \\
\text { Naltrexona }(100) \\
\text { Sertralina }(200) \\
\text { +Naltrexona }(100) \\
\text { Placebo }\end{array}$ & $\begin{array}{l}\text { DSM-IV-R/ SCID } \\
\text { + cuestionario } \\
\text { para diferenciar } \\
\text { inducidos de } \\
\text { primarios }\end{array}$ & $\begin{array}{c}40 \\
49 \\
42 \\
39 \\
\text { Placebo }\end{array}$ & $\begin{array}{c}12 \text { o más bebidas } \\
\text { alcohólicas por } \\
\text { semana }\end{array}$ & 14 & TCC semanal & $\begin{array}{l}\text { TLFB: La combinación sertralina + naltrexona } \\
\text { produjo una tasa de abstinencia de alcohol } \\
\text { más alta }(53,7 \% ; p=0,001 ; O R=3,7) \text {, que } \\
\text { los otros grupos de tratamiento: naltrexona } \\
(21,3 \%) \text {, sertralina }(27,5 \%) \text {, o placebo } \\
(23,1 \%) \\
\text { HAMD: los pacientes en sertralina + naltrexona } \\
\text { estaban menos deprimidos al final del } \\
\text { tratamiento }(83,3 \% ; p=, 014 ; 0 R=3,6) \text {, en } \\
\text { comparación con los otros grupos. }\end{array}$ \\
\hline
\end{tabular}

Nota. BDI: Beck Depression Inventary; DSM-IV: Manual diagnóstico y estadístico de los trastornos mentales; ECA: Ensayo clínico aleatorizado; HAMD: Escala Hamilton depression; K-SADS-PL: The Schedule for Affective Disorders and Schizophrenia for School-Age Children-Present and Lifetime version; MADRS: Montgomery-Asberg Depression Rating Scale; OR: Odds Ratio; PRISM: Psychiatric Research Interview for Substance Use Disorders; SCID: Structured Clinical Interview for DSM Disorders; TCC: Terapia cognitivo-conductual; TLFB: Time line follow back.

(evidencia de calidad moderada). En tres ECA (Cornelius et al., 1997; Gual et al., 2003; Kranzler et al., 2006) tampoco se encontraron diferencias con respecto al tiempo hasta la recaída $(\mathrm{n}=163)$ (evidencia de muy baja calidad).
Cuatro ECA evaluaron variables pragmáticas, como el abandono del tratamiento debido a efectos secundarios, utilizando el instrumento de Evaluación Sistemática Modificada para Tratamiento y Eventos Emergentes (SAFTEE) y 
la retención del tratamiento (Gual et al., 2003; Moak et al., 2003; Pettinati et al., 2010; Roy, 1998). En un ECA (Pettinati et al., 2010) no se encontraron diferencias entre ISRS y placebo en la puntuación SAFTEE $(n=79)$ (evidencia de baja calidad). Respecto a la retención del tratamiento evaluada en tres ECA (Gual et al., 2003; Moak et al., 2003; Roy, 1998), no se observaron diferencias entre los ISRS y el placebo $(\mathrm{n}=201)$ (evidencia de calidad moderada).

\section{- Recomendaciones}

- La administración de antidepresivos ISRS para mejorar los síntomas depresivos no se recomienda (recomendación fuerte).

- La administración de antidepresivos SSRI para reducir el consumo de alcohol no se recomienda (recomendación fuerte). No se puede hacer ninguna recomendación para el resultado «tiempo de recaída» (recomendación débil).

- Respecto de las variables pragmáticas (retención del tratamiento y abandono del tratamiento debido a efectos secundarios), no se pueden hacer recomendaciones (recomendación débil).

Pregunta 2 de PICO. ¿Es eficaz la administración de antidepresivos distintos de los ISRS para reducir los síntomas de la depresión, reducir el consumo de alcohol o mejorar las variables pragmáticas y de funcionalidad en pacientes con depresión y consumo de alcohol?

Se incluyeron cinco estudios en la revisión, dos de ellos evaluando la eficacia de los antidepresivos tricíclicos (Mason, Kocsis, Ritvo y Cutler, 1996; McGrath et al., 1996), uno la viloxazina (Altamura, Mauri, Girardi y Panetta, 1990) y dos evaluando nefazodona (Hernandez-Avila, Modesto-Lowe, Feinn y Kranzler, 2004; Roy-Byrne et al., 2000).

Tres ECA evaluaron la eficacia de antidepresivos distintos de los ISRS frente a placebo para reducir los síntomas depresivos utilizando la escala HAMD (Mason et al., 1996; McGrath et al., 1996; Roy-Byrne et al., 2000) y mostraron diferencias significativas a favor del tratamiento activo a las 12 semanas $(n=107)$, con la mayor eficacia para la desipramina (Mason et al., 1996) (evidencia de baja calidad).

Cuatro ECA estudiaron la reducción del consumo de alcohol como resultado de interés utilizando el TFLB (Hernandez-Avila et al., 2004; Mason et al., 1996; McGrath et al., 1996; Roy-Byrne et al., 2000). A las 12 semanas, no se encontraron diferencias entre los antidepresivos distintos de los ISRS y el placebo $(\mathrm{n}=150)$ (evidencia de calidad moderada).

Tres ECA compararon otros depresores con placebo respecto a las variables pragmáticas (Hernandez-Avila et al., 2004; Mason et al., 1996; Roy-Byrne et al., 2000). En dos ECA (Mason et al., 1996; Roy-Byrne et al., 2000), no se observaron diferencias en la puntuación SAFTEE o efectos secundarios autoinformados $(\mathrm{n}=86)$ (evidencia de calidad moderada). La retención del tratamiento se evaluó en dos
ECA (Hernandez-Avila et al., 2004; Roy-Byrne et al., 2000) y las diferencias entre otros antidepresivos y placebo no fueron significativas $(\mathrm{n}=105)$ (evidencia de baja calidad).

\section{- Recomendaciones}

- En pacientes con depresión y consumo de alcohol, se recomienda la administración de antidepresivos no ISRS, principalmente antidepresivos tricíclicos (recomendación fuerte).

- La administración de antidepresivos no ISRS para reducir el consumo de alcohol no se recomienda (recomendación débil).

- No se pueden hacer recomendaciones respecto del efecto de los antidepresivos no SRRI sobre las variables pragmáticas (abandono del tratamiento debido a efectos secundarios y el tratamiento de retención) (recomendación débil).

\section{Pacientes con trastorno depresivo y consumo de cannabis}

Los detalles sobre los estudios incluidos se muestran en la Tabla 3 .

Pregunta 3 de PICO. ¿Es eficaz la administración de antidepresivos distintos de los ISRS para reducir los síntomas de la depresión, reducir el consumo de cannabis o mejorar las variables pragmáticas y de funcionalidad en pacientes con depresión y consumo de cannabis?

Un ECA evaluó el efecto de la venlafaxina sobre el resultado de la reducción de los síntomas depresivos a las 12 semanas mediante la escala HAMD (Levin et al., 2013) y no se encontraron diferencias $(n=103)$ (evidencia de calidad moderada). Este ECA (Levin et al., 2013) también evaluó el efecto de la venlafaxina para reducir el consumo de cannabis evaluado por los niveles cuantitativos de tetrahidrocannabinol (THC) en orina. A las 12 semanas, se observaron diferencias a favor del placebo $(n=103)$ (evidencia de calidad moderada).

El mismo ECA (Levin et al., 2013) evaluó variables pragmáticas y no se encontraron diferencias entre los antidepresivos no SRRI y el placebo en los resultados del abandono del tratamiento a las 12 semanas debido a efectos secundarios $(\mathrm{n}=103)$ (baja calidad de evidencia) y retención del tratamiento $(\mathrm{n}=103)$ (evidencia de calidad moderada).

\section{- Recomendaciones}

- En pacientes con depresión y consumo de cannabis, no se recomienda la administración de venlafaxina para reducir los síntomas depresivos (recomendación débil).

- No se recomienda el uso de venlafaxina para reducir el consumo de cannabis (recomendación débil).

- No es posible hacer ninguna recomendación sobre el efecto de la venlafaxina sobre las variables pragmáticas (abandono del tratamiento debido a efectos 
Tabla 3. Depresión y trastorno por uso de cannabis.

\begin{tabular}{|c|c|c|c|c|c|c|c|}
\hline AUTORES & $\begin{array}{c}\text { FÁRMACO } \\
\text { UTILIZADO } \\
\text { MG/DIA }\end{array}$ & $\begin{array}{l}\text { DIAGNÓSTICO/ } \\
\text { INSTRUMENTO }\end{array}$ & $\begin{array}{c}\text { CASO/ } \\
\text { CONTROL }\end{array}$ & $\begin{array}{l}\text { CONSUMO AL } \\
\text { INICIO DEL ECA }\end{array}$ & SEMANAS & $\begin{array}{c}\text { TERAPIA } \\
\text { CONCOMITANTE }\end{array}$ & VARIABLE DE RESULTADO \\
\hline
\end{tabular}

Nota. CO: Controles de Orina; DSM-IV: Manual diagnóstico y estadístico de los trastornos mentales; HAMD: Escala Hamilton Depresion; OR: Odds Ratio; SCID: Structured Clinical Interview for DSM Disorders; TCC: Terapia cognitivo-conductual; TLFB: Time line follow back; VEN-XR: Venlafaxina de liberación extendida.

secundarios y retención del tratamiento) (recomendación débil).

\section{Pacientes con trastorno depresivo y consumo de cocaína}

Los detalles sobre los estudios incluidos se muestran en la Tabla 4.

Pregunta 4 de PICO. ¿La administración de antidepresivos ISRS es eficaz para reducir los síntomas de depresión, para reducir el consumo de cocaína o para mejorar las variables pragmáticas y funcionales en pacientes con depresión y consumo de cocaína?

Un ECA evaluó la mejoría de los síntomas depresivos a las 12 semanas mediante la escala HAMD (Schmitz et al., 2001) y no hubo diferencias significativas entre los antidepresivos ISRS y el placebo $(n=68)$ (evidencia de baja calidad).

Tres ECA (Mancino et al., 2014; Oliveto et al., 2012; Schmitz et al., 2001) compararon los antidepresivos ISRS con el placebo para el resultado de disminución del consumo de cocaína a las 12 semanas mediante análisis de cocaína en orina y no se encontraron diferencias significativas $(\mathrm{n}=$ 177) (evidencia de baja calidad).

Un ECA (Schmitz et al., 2001) comparó los antidepresivos ISRS con el placebo para la retención del tratamiento y no se encontraron diferencias $(n=68)$ (evidencia de muy baja calidad).

\section{- Recomendaciones}

- En pacientes con depresión y consumo de cocaína, no se recomienda el uso de antidepresivos ISRS para mejorar los síntomas depresivos (recomendación débil). No se pueden hacer recomendaciones respecto al uso de antidepresivos no ISRS.

- No se recomienda la administración de antidepresivos ISRS para reducir el consumo de cocaína (recomendación fuerte).

- No se recomienda la administración de antidepresivos ISRS para mejorar la retención del tratamiento (recomendación débil).
Pregunta 5 de PICO. ¿Es eficaz la administración de antidepresivos distintos de los ISRS para reducir los síntomas de la depresión, reducir el consumo de cocaína o mejorar las variables pragmáticas y de funcionalidad en pacientes con depresión y consumo de cocaína?

Un total de seis ECA evaluaron la eficacia de antidepresivos no ISRS, tres de ellos evaluaron antidepresivos tricíclicos (McDowell et al., 2005; Nunes et al., 1995; Ziedonis y Kosten, 1991); uno nefazodona (Ciraulo et al., 2005); uno mirtazapina (Afshar et al., 2012); y uno venlafaxina (Raby et al., 2014)

Tres ECA (McDowell et al., 2005; Nunes et al., 1995; Raby et al., 2014) evaluaron el efecto de los antidepresivos no ISRS frente a placebo en la reducción de los síntomas depresivos a las 12 semanas usando HDMA. Se encontraron diferencias a favor del tratamiento activo $(\mathrm{n}=310)$, siendo la desipramina la intervención más eficaz (Nunes et al., 1995) (evidencia de calidad moderada). En relación a la gravedad de la depresión evaluada con la escala de impresión clínica global (CGI), no se encontraron diferencias entre los antidepresivos no ISRS y el placebo $(n=259)$ (evidencia de baja calidad).

Cinco ECA evaluaron el resultado de la reducción del consumo de cocaína. En cuatro ECA (McDowell et al., 2005; Nunes et al., 1995; Raby et al., 2014; Ziedonis y Kosten, 1991), no se encontraron diferencias mediante una analítica de orina entre los antidepresivos no ISRS y el placebo en la disminución del consumo de cocaína a las 12 semanas $(\mathrm{n}=324)$ (evidencia de calidad moderada). Además, en dos ECA (Afshar et al., 2012; McDowell et al., 2005) no se encontraron diferencias significativas en el craving de cocaína utilizando la escala Conceptual Craving Scale $(\mathrm{CCS}) \quad(\mathrm{n}=129)$ (evidencia de muy baja calidad). Para el resultado «días de la semana consumiendo cocaína» analizado en tres ECA (Afshar et al., 2012; McDowell et al., 2005; Raby et al., 2014), no se observaron diferencias significativas $(\mathrm{n}=259)$ (evidencia de calidad moderada).

El abandono del tratamiento debido a efectos secundarios se evaluó en tres ECA (McDowell et al., 2005; Nunes et al., 1995; Raby et al., 2014), y no hubo diferencias signi- 
Guía de práctica clínica para el tratamiento farmacológico y psicológico de los pacientes adultos con depresión y un diagnóstico comórbido de trastorno por uso de sustancias

Tabla 4. Depresión y trastorno por uso de cocaína.

\begin{tabular}{|c|c|c|c|c|c|c|c|}
\hline AUTORES & $\begin{array}{l}\text { FÁRMACO } \\
\text { UTILIZADO } \\
\text { MG/DIA }\end{array}$ & $\begin{array}{l}\text { DIAGNÓSTICO/ } \\
\text { INSTRUMENTO }\end{array}$ & $\begin{array}{l}\text { CASO/ } \\
\text { CONTROL }\end{array}$ & $\begin{array}{l}\text { CONSUMO AL } \\
\text { INICIO DEL ECA }\end{array}$ & SEMANAS & $\begin{array}{c}\text { TERAPIA } \\
\text { CONCOMITANTE }\end{array}$ & VARIABLE DE RESULTADO \\
\hline $\begin{array}{l}\text { Nunes } \\
1995\end{array}$ & $\begin{array}{l}\text { Imipramina } \\
(150-300) \\
\text { Placebo }\end{array}$ & DSM-III-R/SCID & $38 / 31$ & Consumo actual & 12 & $\begin{array}{l}\text { Counselling } \\
\text { Individual }\end{array}$ & $\begin{array}{l}\text { C0: aumento no significativo de CO negativos durante } \\
\text { tres semanas consecutivas en imipramina ( } 26 \%) \text { vs. } \\
\text { Placebo (13\%). } \\
\text { HDRS: Sin efecto en el tratamiento de la depresión. }\end{array}$ \\
\hline $\begin{array}{l}\text { Cornelius } \\
1998\end{array}$ & $\begin{array}{l}\text { Fluoxetina (20) } \\
\text { Placebo }\end{array}$ & DSM-III-R/SCID & $8 / 9$ & $\begin{array}{l} \pm 9 \text { días de } \\
\text { abstinencia }\end{array}$ & 12 & Terapia de apoyo & $\begin{array}{l}\text { CO, ASI, TLFB, AR: No se observaron diferencias } \\
\text { significativas en el consumo de cocaína intra o } \\
\text { intergrupos. } \\
\text { BDI: La puntuación media en BDI empeoró } 2,2 \text { puntos } \\
\text { en Placebo y mejoró 3,9 puntos en fluoxetina, sin } \\
\text { significación estadística. }\end{array}$ \\
\hline $\begin{array}{l}\text { Schmitz } \\
2001\end{array}$ & $\begin{array}{l}\text { Fluoxetina (40) } \\
\text { Placebo }\end{array}$ & DSM-IV/SCID & $34 / 34$ & Consumo actual & 12 & $\begin{array}{l}\text { TCC y prevención de } \\
\text { recaídas }\end{array}$ & $\begin{array}{l}\text { CO: No diferencias significativas en CO negativos al } \\
\text { final del tratamiento entre ambos grupos. } \\
\text { HAMD: Los síntomas depresivos remiten como una } \\
\text { función del tiempo en tratamiento, sin diferencias } \\
\text { significativas. }\end{array}$ \\
\hline $\begin{array}{l}\text { Mc Dowell } \\
2005\end{array}$ & $\begin{array}{l}\text { Desipramina } \\
(300) \\
\text { Placebo }\end{array}$ & $\begin{array}{l}\text { DSM-III-R /SCID } \\
\text { consulta con } 2 \\
\text { expertos }\end{array}$ & $55 / 56$ & Consumo actual & 12 & $\begin{array}{l}\text { TCC y prevención de } \\
\text { recaídas }\end{array}$ & $\begin{array}{l}\text { TLFB, CO: Los grupos de tratamiento no mostraron } \\
\text { diferencias en la tasa de respuesta. } \\
\text { CGI, HAMD: Desipramina se asoció con una mayor } \\
\text { tasa de respuesta en los síntomas depresivos }(51 \% \text {, } \\
28 / 55) \text { que el placebo }(32 \%, 18 / 56)(p<0,05) \text {. }\end{array}$ \\
\hline $\begin{array}{l}\text { Asphar } \\
2012\end{array}$ & $\begin{array}{l}\text { Mirtazapina } \\
\text { (45) Placebo }\end{array}$ & DSM-IV & $11 / 13$ & Consumo actual & 12 & $\begin{array}{l}\text { Prevención de } \\
\text { recaídas }\end{array}$ & $\begin{array}{l}\text { CO / AR: El consumo de cocaína durante el periodo } \\
\text { de tratamiento no difirió significativamente entre los } \\
\text { grupos mirtazapina y placebo. } \\
\text { HAMD: Reducción significativa en la semana } 1 \text { en las } \\
\text { puntuaciones HAMD, tanto para la mirtazapina [ } p= \\
0,002 \text { ] como para el grupo placebo }[p=0,005] \text {. }\end{array}$ \\
\hline $\begin{array}{l}\text { Oliveto } \\
2012\end{array}$ & $\begin{array}{l}\text { Sertralina (200) } \\
\text { Placebo }\end{array}$ & DSM-IV (SCID) & $32 / 27$ & Consumo actual & 12 & TCC & $\begin{array}{l}\text { CO/AR: } 19(70,3 \%) \text { placebo y } 17(53,1 \%) \text { sertralina } \\
\text { experimentaron una recaída en el consumo. } \\
\text { HAMD: Las puntuaciones disminuyeron } \\
\text { significativamente con el tiempo }(p<0,0001) \text {, pero sin } \\
\text { diferencias entre los grupos }(p=0,77) \text {. }\end{array}$ \\
\hline $\begin{array}{l}\text { Mancino } \\
2014\end{array}$ & $\begin{array}{l}\text { Sertralina (200) } \\
\text { Placebo }\end{array}$ & DSM-IV (SCID) & $23 / 27$ & Consumo actual & 12 & TCC & $\begin{array}{l}\text { CO: La sertralina mostró un porcentaje global } \\
\text { significativamente menor de muestras de orina } \\
\text { positivas en comparación con placebo. } \\
\text { HAMD: las puntuaciones de depresión } \\
\text { disminuyeron significativamente con el tiempo } \\
\text { independientemente del grupo de tratamiento. }\end{array}$ \\
\hline $\begin{array}{l}\text { Raby } \\
2014\end{array}$ & $\begin{array}{l}\text { Venlafaxina } \\
(300) \\
\text { Placebo }\end{array}$ & DSM-IIIR/ SCID & $66 / 66$ & Consumo actual & 12 & $\begin{array}{l}\text { Prevención de } \\
\text { recaídas }\end{array}$ & $\begin{array}{l}\text { CO: No se encontraron diferencias entre los grupos } \\
\text { de tratamiento. La proporción de pacientes que } \\
\text { alcanzaron tres o más semanas consecutivas } \\
\text { de abstinencia confirmadas en orina fue baja } \\
\text { (venlafaxina: } 16 \% \text {; placebo: } 15 \%) \text {. } \\
\text { HAMD: La mejoría del estado de ánimo fue del } 41 \% \\
\text { (26/64) en el grupo venlafaxina, y el } 33 \%(22 / 66) \text { en } \\
\text { el grupo placebo. }\end{array}$ \\
\hline
\end{tabular}

Nota. AR: Consumo de cocaína auto informado; ASI:addiction severity index; BDI: Beck Depression Inventary; CGI: Clinical Global Impression Scale; CO: control de orina; DSM-IV: Manual diagnóstico y estadístico de los trastornos mentales; ECA: Ensayo clínico aleatorizado; HAMD: Hamilton depression rating Scale; MC: Manejo de contingencias; PMM: Programa de mantenimiento con metadona; SCID: Structured Clinical Interview for DSM Disorders; TCC: Terapia Cognitivo Conductual;. TLFB: time-line follow-back. 
ficativas entre los antidepresivos no ISRS y el placebo (n =354) (evidencia de muy baja calidad). En relación a la retención del tratamiento evaluada en dos ECA (McDowell et al., 2005; Raby et al., 2014), no se encontraron diferencias $(\mathrm{n}=241)$ (evidencia de baja calidad).

\section{- Recomendaciones}

- Se recomienda el uso de antidepresivos distintos de los ISRS, principalmente antidepresivos tricíclicos, para mejorar los síntomas depresivos (recomendación fuerte).

- No se recomienda el uso de antidepresivos distintos de los ISRS para reducir el consumo de cocaína (abstinencia) (recomendación fuerte).

- No se recomienda el uso de antidepresivos distintos de los ISRS para reducir el craving (recomendación débil).

- No se recomienda el uso de antidepresivos distintos de los ISRS para mejorar la retención del tratamiento o reducir el abandono del tratamiento debido a efectos secundarios (recomendación débil).

\section{Pacientes con trastorno depresivo y uso de nicotina}

Los detalles sobre los estudios incluidos se muestran en la Tabla 5.

Pregunta 6 de PICO. ¿La administración de antidepresivos distintos de los ISRS es eficaz para reducir el consumo de nicotina en pacientes con depresión y consumo de nicotina?
Tres ECA han comparado la efectividad de los antidepresivos no ISRS (bupropión) frente a placebo para el cese del tabaquismo medido por los niveles de monóxido de carbono exhalado (Catley et al., 2005; Evins et al., 2008; Schnoll et al., 2010). No se hallaron diferencias entre los dos grupos del estudio $(\mathrm{n}=306)$ (evidencia de calidad moderada).

\section{- Recomendaciones}

- No se recomienda la administración de los antidepresivos no ISRS (bupropión) para reducir el consumo de nicotina (recomendación fuerte).

\section{Tratamiento psicológico}

Pregunta 7 de PICO. ¿Es eficaz el tratamiento psicológico para reducir los sintomas depresivos o para reducir el consumo de alcohol en pacientes con depresión y consumo de alcohol?

El metaanálisis de Hobbs et al. (Hobbs, Kushner, Lee, Reardon y Maurer, 2011) informa de los efectos de 15 ensayos ECA publicados que examinaron el impacto de complementar el tratamiento para el trastorno por uso de alcohol con tratamiento farmacológico frente a terapia cognitivo-conductual (TCC) para la mejora de los síntomas de internalización concurrentes (ansiedad o depresión). La intervención de TCC tuvo una estimación del tamaño del efecto agrupado de Cohen $d=0,66$, mientras que la medicación produjo una estimación más baja del tamaño

Tabla 5. Depresión y trastorno por uso de nicotina.

\begin{tabular}{|c|c|c|c|c|c|c|c|}
\hline AUTORES & $\begin{array}{l}\text { FÁRMACO } \\
\text { UTILIZADO } \\
\text { MG/DIA }\end{array}$ & $\begin{array}{l}\text { DIAGNÓSTICO/ } \\
\text { INSTRUMENTO }\end{array}$ & $\begin{array}{l}\text { CASO/ } \\
\text { CONTROL }\end{array}$ & $\begin{array}{l}\text { CONSUMO AL } \\
\text { INICIO DEL ECA }\end{array}$ & SEMANAS & $\begin{array}{c}\text { TERAPIA } \\
\text { CONCOMITANTE }\end{array}$ & VARIABLE DE RESULTADO \\
\hline $\begin{array}{l}\text { Brown } \\
2007\end{array}$ & $\begin{array}{l}\text { Bupropion (150) } \\
\text { Placebo }\end{array}$ & CES-D & $108 / 157$ & Consumo actual & 12 & TCC & $\begin{array}{l}\text { CMC: Bupropion mostró mejores resultados para } \\
\text { fumadores en ambos tratamientos grupales } \\
\text { intensivos. }\end{array}$ \\
\hline $\begin{array}{l}\text { Catley } \\
2005\end{array}$ & $\begin{array}{l}\text { Bupropion (150) } \\
\text { Placebo }\end{array}$ & CES-D & $78 / 83$ & Consumo actual & 7 & $\begin{array}{l}\text { Counselling y } \\
\text { guia para dejar } \\
\text { de fumar }\end{array}$ & $\begin{array}{l}\text { CMC: (autoinformado/ CO): No se encontraron } \\
\text { diferencias significativas entre los grupos. El grupo } \\
\text { placebo disminuye en mayor medida el consumo de } \\
\text { nicotina. }\end{array}$ \\
\hline $\begin{array}{l}\text { Evins } \\
2008\end{array}$ & $\begin{array}{l}\text { Bupropion (150)+ } \\
\text { NRT (21) / } \\
\text { Placebo }\end{array}$ & DSM-IV/SCID & $45 / 45$ & Consumo actual & 13 & TCC & $\begin{array}{l}\text { TLFB/ CO: Las tasas de abstinencia al final del ensayo } \\
\text { fueron } 36 \%(37 / 97) \text { en el grupo bupropion + NRT + } \\
\text { TCC y el 31\% (32/102) en el grupo placebo + NRT + } \\
\text { TCC. No estadísticamente significativas. }\end{array}$ \\
\hline $\begin{array}{l}\text { Schnoll } \\
2010\end{array}$ & $\begin{array}{l}\text { Bupropion + NRT / } \\
\text { placebo. }\end{array}$ & CES-D & $28 / 27$ & Consumo actual & 9 & Counseling + NRT & $\begin{array}{l}\text { CMC: No hubo un efecto principal de bupropion } \\
\text { frente a placebo en la abstinencia (OR 1,36). Los } \\
\text { pacientes con síntomas de depresión informaron } \\
\text { significativamente menores tasas de abstinencia } \\
\text { frente a los pacientes sin síntomas de depresión (OR } \\
=0,14) \text {. }\end{array}$ \\
\hline
\end{tabular}

Nota. BDI: Beck Depression Inventory; CES-D: Center for Epidemiologic Studies Depression Scale; CMC: Concentración Monóxido de Carbono; CO: Monóxido de carbono; DSM-IV: Manual diagnóstico y estadístico de los trastornos mentales; ECA: Ensayo clínico aleatorizado; NRT parche transdérmico; OR: Odds ratio; SCID; Structured Clinical Interview for DSM Disorders; TCC: Terapia Cognitivo Conductual; TLFB: time-line follow-back.

${ }^{\star *}$ Análisis secundarios. 
del efecto agrupado de $d=0,24$ (evidencia de muy baja calidad). El metaanálisis de Riper et al. (2014) realizado en 12 estudios evaluó la efectividad de combinar la TCC y la entrevista motivacional (EM) (TCC/EM) para tratar el trastorno por consumo de alcohol concomitante y la depresión mayor en comparación con la atención habitual en un total de 1721 pacientes (grupo experimental 1026, grupo de control 695). Los efectos de la TCC/EM sobre la disminución de los síntomas de depresión en los controles fueron pequeños pero significativos en la posprueba ( $g$ de Hedge $=0,27$; IC del $95 \% 0,13$ a 0,$41 ; p<, 001)$. Cuando se evaluó el impacto de la TCC/EM sobre los síntomas depresivos durante el seguimiento (6 a 12 meses después del tratamiento), se encontró un tamaño del efecto similar ( $g=0,26$; IC del $95 \%-0,01$ a 0,54 ) (evidencia de calidad moderada).

En el estudio de Hobbs et al. (2011) con un total de tres estudios para el análisis del resultado del alcohol, el tamaño del efecto para la disminución del consumo de alcohol fue mayor para la TCC $(d=0,29)$ que para el tratamiento farmacológico $(d=0,17)$ pero las diferencias no fueron significativas (evidencia de baja calidad). En el metaanálisis de Riper et al. (2014), TCC/EM mostró un efecto pequeño pero significativo en la reducción del consumo de alcohol $(g=0,17)$. A los 6-12 meses de seguimiento, el efecto fue $g$ $=0,31$ (evidencia de calidad moderada).

\section{- Recomendaciones}

- En pacientes con depresión y trastorno por consumo de alcohol concurrente, tanto la farmacoterapia como la terapia cognitivo-conductual tienen efectos positivos sobre los síntomas de internalización (niveles de ansiedad y depresión) (recomendación débil).

- En pacientes con depresión y trastorno por consumo de alcohol concurrente, tanto la farmacoterapia como la terapia cognitivo-conductual tienen efectos positivos para reducir el consumo de alcohol (recomendación débil).

Pregunta 8 de PICO. ¿Es eficaz el tratamiento psicológico para reducir los sintomas depresivos o para reducir el uso de sustancias en pacientes con depresión y trastorno por uso de sustancias?

Hesse et al. (2009) realizaron una revisión sistemática y un metaanálisis para evaluar el tratamiento integrado de los trastornos por uso de sustancias y la depresión comórbida en comparación con un programa de tratamiento centrado únicamente en el trastorno por uso de sustancias (control). Para el resultado de mejoría de los síntomas depresivos evaluados con la Escala de Calificación de Hamilton para la Depresión (HRSD), el efecto combinado fue $d$ $=-4,6$ puntos en la HRSD para la condición experimental en comparación con el control (IC del $95 \%$-7,4 a 1,7), con una heterogeneidad moderadamente alta significativa en el resultado $\left(I^{2}=0,61, p=, 05\right)$ (evidencia de baja calidad). Para los estudios que informaron resultados del cuestiona- rio de autoinforme para la depresión, el efecto combinado fue $d=-0,58$ (IC del $95 \%-1,10$ a -0,06). La heterogeneidad no fue significativa y de baja a moderada $\left(I^{2}=0,46, p\right.$ $=, 14)$ (evidencia de baja calidad). Respecto del resultado del porcentaje de días de abstinencia, la comparación favoreció el tratamiento con una diferencia media de 13,75 (IC del $95 \% 0,51$ a 22,99) $\left(I^{2}=0,17, p=, 30\right)$ (evidencia de calidad moderada).

\section{- Recomendaciones}

- La terapia psicológica para la depresión comórbida y el TUS es un enfoque prometedor, pero no está lo suficientemente respaldado empíricamente como una opción para mejorar los síntomas depresivos (recomendación moderada).

\section{Conclusiones}

Esta revisión evidencia que se ha realizado un pequeño número de ensayos doble ciego y controlados con placebo en pacientes con depresión y TUS con el objetivo de evaluar el impacto de la farmacoterapia tanto en los síntomas depresivos como en la reducción del consumo de sustancias. Varios ensayos clínicos han demostrado un efecto beneficioso de los antidepresivos sobre los síntomas del estado de ánimo en pacientes con TUS comórbido, pero aún no han podido establecer su eficacia sobre los resultados en el uso de sustancias debido a resultados inconsistentes.

Nuestros resultados sugieren que 1) en pacientes con depresión y trastorno por consumo de alcohol, se recomienda la administración de antidepresivos no ISRS en lugar de ISRS para mejorar los síntomas depresivos (recomendación fuerte). No se recomiendan antidepresivos ISRS (recomendación fuerte) ni antidepresivos no ISRS (recomendación débil) para reducir el consumo de alcohol; 2) en pacientes con depresión y trastorno por consumo de cannabis, no se recomienda el uso de venlafaxina (recomendación débil); 3) en pacientes con depresión y trastorno por consumo de cocaína, no se recomienda el uso de antidepresivos ISRS para mejorar los síntomas depresivos (recomendación débil) o para reducir el consumo de cocaína (recomendación fuerte). El uso de antidepresivos no ISRS solo se recomienda para mejorar los síntomas depresivos (recomendación fuerte); 4) no se recomienda la administración de bupropión para reducir el consumo de nicotina (recomendación fuerte); 5) respecto del tratamiento psicológico, en pacientes con depresión y trastorno por consumo de alcohol concurrente, tanto la farmacoterapia como la terapia cognitivo-conductual tienen efectos positivos en la internalización de los síntomas y en la reducción del consumo de alcohol (recomendación débil).

Muy pocos de los ensayos aleatorios realizados hasta la fecha han proporcionado evidencia consistente para el manejo tanto de la depresión como del uso de sustancias. En pacientes con depresión y consumo de cannabis, solo se ha 
analizado la venlafaxina. Por tanto, es obligatoria la realización de más investigaciones en esta área y de estudios aleatorizados, multisitio con muestras generalizables para proporcionar evidencia más definitiva.

\section{Reconocimientos}

Servicio Gallego de Salud (SERGAS) y Sociedad Española de Psiquiatría Biológica (SEPB).

\section{Conflicto de intereses}

Los autores declaran la inexistencia de conflicto de interés alguno en relación a este artículo.

\section{Referencias}

Afshar, M., Knapp, C. M., Sarid-Segal, O., Devine, E., Colaneri, L. S., Tozier, L.,... Ciraulo, D. A. (2012). The efficacy of mirtazapine in the treatment of cocaine dependence with comorbid depression. American Journal of Drug and Alcohol Abuse, 38, 181-186. doi:10.3109/00952990. 2011.644002.

Agabio, R., Trogu, E. y Pani, P. P. (2018). Antidepressants for the treatment of people with co-occurring depression and alcohol dependence. The Cochrane Database of Systematic Reviews, 4, CD008581. doi:10.1002/14651858. CD008581.pub2.

Alías-Ferri, M., García-Marchena, N., Mestre-Pintó, J. I., Araos, P., Vergara-Moragues, E., Fonseca, F.,... Torrens, M. (2021). Cocaine and depressive disorders: When standard clinical diagnosis is insufficient. Adicciones, 33, 193-200. doi:10.20882/adicciones.1321.

Altamura, A. C., Mauri, M. C., Girardi, T. y Panetta, B. (1990). Alcoholism and depression: A placebo controlled study with viloxazine. International Journal of Clinical Pharmacology Research, 10, 293-298.

Boschloo, L., Vogelzangs, N., Smit, J. H., van den Brink, W., Veltman, D. J., Beekman, A. T. y Penninx, B. W. (2011). Comorbidity and risk indicators for alcohol use disorders among persons with anxiety and/or depressive disorders: Findings from the Netherlands Study of Depression and Anxiety (NESDA). Journal of Affective Disorders, 131, 233-242. doi:10.1016/j.jad.2010.12.014.

Caetano, R., Vaeth, P. A. C. y Canino, G. (2019). Comorbidity of lifetime alcohol use disorder and major depressive disorder in San Juan, Puerto Rico. Journal of Studies on Alcohol and Drugs, 80, 546-551. doi:10.15288/ jsad.2019.80.546.

Carey, T. L. (2019). Use of antidepressants in patients with co-occurring depression and substance use disorders. Handbook of Experimental Pharmacology, 250, 359-370. doi:10.1007/164_2018_162.
Carton, L., Pignon, B., Baguet, A., Benradia, I., Roelandt, J.-L., Vaiva, G.,... Rolland, B. (2018). Influence of comorbid alcohol use disorders on the clinical patterns of major depressive disorder: A general population-based study. Drug and Alcohol Dependence, 187, 40-47. doi:10.1016/j.drugalcdep.2018.02.009.

Catley, D., Harris, K. J., Okuyemi, K. S., Mayo, M. S., Pankey, E. y Ahluwalia, J. S. (2005). The influence of depressive symptoms on smoking cessation among African Americans in a randomized trial of bupropion. Nicotine $\mathcal{E}$ Tobacco Research : Official Journal of the Society for Research on Nicotine and Tobacco, 7, 859-870. doi:10.1080/14622200500330118.

Ciraulo, D., Knapp, C., Rotrosen, J., Sarid-Segal, O., Ciraulo, A. M., LoCastro, J.,... Leiderman, D. (2005). Nefazodone treatment of cocaine dependence with comorbid depressive symptoms. Addiction, 100 (Supl. 1), 23-31. doi:10.1111/j.1360-0443.2005.00984.x.

Compton, W. M., Thomas, Y. F., Stinson, F. S. y Grant, B. F. (2007). Prevalence, correlates, disability, and comorbidity of DSM-IV alcohol abuse and dependence in the United States: Results from the National Epidemiologic Survey on Alcohol and Related Conditions. Archives of General Psychiatry, 64, 830-842. doi.10.1001/ archpsyc.64.5.566.

Conner, K. R., Pinquart, M. y Holbrook, A. P. (2008). Meta-analysis of depression and substance use and impairment among cocaine users. Drug and Alcohol Dependence, 98, 13-23. doi:10.1016/j.drugalcdep.2008.05.005.

Conner, K. R., Pinquart, M. y Gamble, S. A. (2009). Meta-analysis of depression and substance use among individuals with alcohol use disorders. Journal of Substance Abuse Treatment, 27, 127-137. doi:10.1016/j. gde.2016.03.011.

Cornelius, J. R., Salloum, I. M., Ehler, J. G., Jarrett, P. J., Cornelius, M. D., Black, A.,... Thase, M. E. (1997). Double-blind fluoxetine in depressed alcoholic smokers. Psychopharmacology Bulletin, 33, 165-170.

Cuenca-Royo, A. M., Torrens, M., Sánchez-Niubó, A., Suelves, J. M. y Domingo-Salvany, A. (2013). Psychiatric morbidity among young-adults cannabis users. Adicciones, 25, 45-53.

Evins, A. E., Culhane, M. A., Alpert, J. E., Pava, J., Liese, B. S., Farabaugh, A. y Fava, M. (2008). A controlled trial of bupropion added to nicotine patch and behavioral therapy for smoking cessation in adults with unipolar depressive disorders. Journal of Clinical Psychopharmacology, 28, 660-666. doi:10.1097/JCP.0b013e31818ad7d6.

Farré, A., Tirado-Muñoz, J. y Torrens, M. (2017). Dual depression: A sex perspective. Addictive Disorders $\mathcal{E}$ Their Treatment, 16, 180-186. doi:10.1097/ ADT.0000000000000118.

Gopalakrishna, G., Langendam, M. W., Scholten, R. J., Bossuyt, P. M. y Leeflang, M. M. (2013). Guidelines for 
guideline developers: A systematic review of grading systems for medical tests. Implementation Science, 8, 78. doi:10.1186/1748-5908-8-78.

Gual, A., Balcells, M., Torres, M., Madrigal, M., Diez, T. y Serrano, L. (2003). Sertraline for the prevention of relapse in detoxicated alcohol dependent patients with a comorbid depressive disorder: A randomized controlled trial. Alcohol and Alcoholism, 38, 619-625. doi:10.1093/ alcalc/agg124.

Guyatt, G. H., Oxman, A. D., Vist, G. E., Kunz, R., Falck-Ytter, Y., Alonso-Coello, P. y Schünemann, H. J. (2008). GRADE: An emerging consensus on rating quality of evidence and strength of recommendations. BMJ, 336, 924-926. doi:10.1136/bmj.39489.470347.AD.

Guyatt, G. H., Oxman, A. D., Kunz, R., Atkins, D., Brozek, J., Vist, G.,... Schünemann, H. J. (2011). GRADE guidelines: 2. Framing the question and deciding on important outcomes. Journal of Clinical Epidemiology, 64, 395-400. doi:10.1016/j.jclinepi.2010.09.012.

Hernandez-Avila, C. A., Modesto-Lowe, V., Feinn, R. y Kranzler, H. R. (2004). Nefazodone treatment of comorbid alcohol dependence and major depression. Alcoholism, Clinical and Experimental Research, 28, 433-440. doi:10.1097/01.ALC.0000118313.63897.EE.

Hesse, M. (2009). Integrated psychological treatment for substance use and co-morbid anxiety or depression vs. treatment for substance use alone. A systematic review of the published literature. BMC Psychiatry, 9, 6. doi:10.1186/1471-244X-9-6.

Hobbs, J. D. J., Kushner, M. G., Lee, S. S., Reardon, S. M. y Maurer, E. W. (2011). Meta-analysis of supplemental treatment for depressive and anxiety disorders in patients being treated for alcohol dependence. American Journal on Addictions, 20, 319-329. doi:10.1111/j.15210391.2011.00140.x.

Hunt, G. E., Malhi, G. S., Lai, H. M. X. y Cleary, M. (2020). Prevalence of comorbid substance use in major depressive disorder in community and clinical settings, 1990-2019: Systematic review and meta-analysis. Journal of Affective Disorders, 266, 288-304. doi:10.1016/j. jad.2020.01.141.

Iqbal, M. N., Levin, C. J. y Levin, F. R. (2019). Treatment for substance use disorder with co-occurring mental illness. Focus, 17, 88-97. doi:10.1176/appi.focus.20180042.

Jiménez-Treviño, L., Velasco, Á., Rodríguez-Revuelta, J., Abad, I., De La Fuente-Tomás, L., González-Blanco, L., ... Sáiz, P. A. (2019). Factors associated with tobacco consumption in patients with depression. Adicciones, 31, 298-308. doi:10.20882/adicciones.1191.

Khantzian, E. J. (1985). The self-medication hypothesis of addictive disorders: Focus on heroin and cocaine dependence. American Journal of Psychiatry, 142, 12591264. doi:10.1176/ajp.142.11.1259.
Kranzler, H. R., Mueller, T., Cornelius, J., Pettinati, H. M., Moak, D., Martin, P. R.,... Keller, M. (2006). Sertraline treatment of co-occurring alcohol dependence and major depression. Journal of Clinical Psychopharmacology, 26, 13-20. doi:10.1097/01.jcp.0000194620.61868.35.

Lai, H. M. X., Cleary, M., Sitharthan, T. y Hunt, G. E. (2015). Prevalence of comorbid substance use, anxiety and mood disorders in epidemiological surveys, 19902014: A systematic review and meta-analysis. Drug and Alcohol Dependence, 1990-2014. doi:10.1016/j.drugalcdep.2015.05.031.

Levin, F. R., Mariani, J., Brooks, D. J., Pavlicova, M., Nunes, E. V., Agosti, V.,... Carpenter, K. M. (2013). A randomized double-blind, placebo-controlled trial of venlafaxine-extended release for co-occurring cannabis dependence and depressive disorders. Addiction, 108, 1084-1094. doi:10.1111/add.12108.

Mancino, M. J., McGaugh, J., Chopra, M. P., Guise, J. B., Cargile, C., Williams, D. K.,... Oliveto, A. (2014). Clinical efficacy of sertraline alone and augmented with gabapentin in recently abstinent cocaine-dependent patients with depressive symptoms. Journal of Clinical Psychopharmacology, 34, 234-239. doi:10.1097/ JCP.0000000000000062.

Mason, B. J., Kocsis, J. H., Ritvo, E. C. y Cutler, R. B. (1996). A double-blind, placebo-controlled trial of desipramine for primary alcohol dependence stratified on the presence or absence of major depression. JAMA, 275, 761767. doi:10.1001/jama.275.10.761.

McDowell, D., Nunes, E. V., Seracini, A. M., Rothenberg, J., Vosburg, S. K., Ma, G. J. y Petkova, E. (2005). Desipramine treatment of cocaine-dependent patients with depression: A placebo-controlled trial. Drug and Alcohol Dependence, 80, 209-221. doi:10.1016/j.drugalcdep.2005.03.026.

McGrath, P. J., Nunes, E. V, Stewart, J. W., Goldman, D., Agosti, V., Ocepek-Welikson, K. y Quitkin, F. M. (1996). Imipramine treatment of alcoholics with primary depression: A placebo-controlled clinical trial. Archives of General Psychiatry, 53, 232-240.

Moak, D. H., Anton, R. F., Latham, P. K., Voronin, K. E., Waid, R. L. y Durazo-Arvizu, R. (2003). Sertraline and cognitive behavioral therapy for depressed alcoholics: results of a placebo-controlled trial. Journal of Clinical Psychopharmacology, 23, 553-562. doi:10.1097/01. jcp.0000095346.32154.41.

Nunes, E. V., McGrath, P. J., Quitkin, F. M., Ocepek-Welikson, K., Stewart, J. W., Koenig, T.,... Klein, D. F. (1995). Imipramine treatment of cocaine abuse: Possible boundaries of efficacy. Drug and Alcohol Dependence, 39, 185195. doi:10.1016/0376-8716\%2895\%2901161-6.

Oliveto, A., Poling, J., Mancino, M. J., Williams, D. K., Thostenson, J., Pruzinsky, R.,... Kosten, T. R. (2012). Sertraline delays relapse in recently abstinent cocaine-depen- 
dent patients with depressive symptoms. Addiction, 107, 131-141. doi:10.1111/j.1360-0443.2011.03552.x.

Pettinati, H. M., Volpicelli, J. R., Luck, G., Kranzler, H. R., Rukstalis, M. R. y Cnaan, A. (2001). Double-blind clinical trial of sertraline treatment for alcohol dependence. Journal of Clinical Psychopharmacology, 21, 143-153. doi:10.1097/00004714-200104000-00005.

Pettinati, H. M., Oslin, D. W., Kampman, K. M., Dundon, W. D., Xie, H., Gallis, T. L.,... O’Brien, C. P. (2010). A double-blind, placebo-controlled trial combining sertraline and naltrexone for treating co-occurring depression and alcohol dependence. American Journal of Psychiatry, 167, 668-675. doi:10.1176/appi.ajp.2009.08060852.

Raby, W. N., Rubin, E. A., Garawi, F., Cheng, W., Mason, E., Sanfilippo, L.,... Nunes, E. V. (2014). A randomized, double-blind, placebo-controlled trial of venlafaxine for the treatment of depressed cocaine-dependent patients. American Journal on Addictions, 23, 68-75. doi:10.1111/ j.1521-0391.2013.12065.x.

Rappeneau, V. y Bérod, A. (2017). Reconsidering depression as a risk factor for substance use disorder: Insights from rodent models. Neuroscience and Biobehavioral Reviews, 77, 303-316. doi:10.1016/j.neubiorev.2017.04.001.

Riper, H., Andersson, G., Hunter, S. B., de Wit, J., Berking, M. y Cuijpers, P. (2014). Treatment of comorbid alcohol use disorders and depression with cognitive-behavioural therapy and motivational interviewing: A meta-analysis. Addiction, 109, 394-406. doi:10.1111/add.12441.

Roy-Byrne, P. P., Pages, K. P., Russo, J. E., Jaffe, C., Blume, A. W., Kingsley, E.,... Ries, R. K. (2000). Nefazodone treatment of major depression in alcohol-dependent patients: A double-blind, placebo-controlled trial. Journal of Clinical Psychopharmacology, 20, 129-136. doi:10.1097/00004714-200004000-00003.

Roy, A. (1998). Placebo-controlled study of sertraline in depressed recently abstinent alcoholics. Biological Psychiatry, 44, 633-637. doi:10.1016/S0006-3223\%2897\%2900509-X.

San, L. y Arranz, B. (2016). Clinical guideline for the treatment of dual pathology in the adult population. Adicciones, 28, 3-5. doi:10.20882/adicciones. 784.

Schmitz, J. M., Averill, P., Stotts, A. L., Moeller, F. G., Rhoades, H. M. y Grabowski, J. (2001). Fluoxetine treatment of cocaine-dependent patients with major depressive disorder. Drug and Alcohol Dependence, 63, 207-214. doi:10.1016/S0376-8716(00)00208-8.

Schnoll, R., Martinez, E., Tatum, K. L., Weber, D. M., Kuzla, N., Glass, M.,... Leone, F. (2010). A bupropion smoking cessation clinical trial for cancer patients. Cancer Causes and Control, 21, 811-820. doi:10.1007/s10552-010-9507-8.

Schuckit, M. A. (2006). Comorbidity between substance use disorders and psychiatric conditions. Addiction, 101 (Supl. 1), 76-88. doi:10.1111/j.1360-0443.2006.01592.x.
Schünemann, H. J., Oxman, A. D., Brozek, J., Glasziou, P., Jaeschke, R., Vist, G. E.,... Guyatt, G. H. (2008). Grading quality of evidence and strength of recommendations for diagnostic tests and strategies. BMJ, 336, 1106-1110. doi:10.1136/bmj.39500.677199.AE.

Sobell, L. y Sobell, M. (1992). Timeline follow-back: A technique for assessing self-reported alcohol consumption. En R. Z. Litten y J. P. Allen (Eds.), Measuring Alcohol Consumption (pp. 41-72). Totowa, NJ: Humana Press.

Tirado-Muñoz, J., Farré, A., Mestre-Pintó, J., Szerman, N. y Torrens, M. (2018). Dual diagnosis in depression: Treatment recommendations. Adicciones, 30, 66-76. doi:10.20882/adicciones.868.

Torrens, M., Fonseca, F., Mateu, G. y Farre, M. (2005). Efficacy of antidepressants in substance use disorders with and without comorbid depression: A systematic review and meta-analysis. Drug and Alcohol Dependence, 78, 1-22. doi:10.1016/j.drugalcdep.2004.09.004.

Torrens, M., Martínez-Sanvisens, D., Martínez-Riera, R., Bulbena, A., Szerman, N. y Ruiz, P. (2011). Dual diagnosis: Focusing on depression and recommendations for treatment. Addictive Disorders $\mathcal{E} \odot$ Their Treatment, 10, 50-59. doi:10.1097/ADT.0b013e318215f322.

Torrens, M., Rossi, P. C., Martinez-Riera, R., Martinez-Sanvisens, D. y Bulbena, A. (2012). Psychiatric co-morbidity and substance use disorders: Treatment in parallel systems or in one integrated system. Substance Use and Misuse, 47, 1005-1014. doi:10.3109/10826084.2012.663296.

Vázquez, F. L., Torres, Á., Otero, P. y Díaz, O. (2011). Prevalence, comorbidity, and correlates of DSM-IV axis I mental disorders among female university students. The Journal of Nervous and Mental Disease, 199, 379-383. doi:10.1097/NMD.0b013e31821cd29c.

Vergara-Moragues, E., González-Saiz, F., M. Lozano, O., Betanzos Espinosa, P., Fernández Calderón, F., Bilbao-Acebos, I.,... Verdejo García, A. (2012). Psychiatric comorbidity in cocaine users treated in therapeutic community: Substance-induced versus independent disorders. Psychiatry Research, 200, 734-741. doi:10.1016/j. psychres.2012.07.043.

Ziedonis, D. M. y Kosten, T. R. (1991). Depression as a prognostic factor for pharmacological treatment of cocaine dependence. Psychopharmacology Bulletin, 27, 337-343. 